\title{
Rebuilding a Teaching Conference in a Pandemic: User-Centered Guiding Principles and Lessons Learned
}

\author{
Laura A. Lukes and E. Shelley Reid
}

\section{Abstract}

The COVID-19 pandemic challenged educational developers, like instructors across the world, to pivot their traditionally face-to-face faculty development programs to online formats. At the Stearns Center for Teaching and Learning at George Mason University (classified as research-intensive and the largest public institution in Virginia, U.S.), we faced the challenge of reimagining our annual pedagogy conference that scaled from 497 registered in 2019 when it was face-to-face to over 800 in 2020 as it was moved online. Under pressures of limited resources and increased uncertainty, leaders can find it difficult to imagine pathways toward innovation rather than just daily responses to crises, yet centers for teaching and learning (CTLs) need to take and maintain leadership roles in our institutions. Here we outline our programmatic goals, our guiding user-centered principles (from user experience frameworks; i.e., Zarour \& Alharbi, 2017), and the user-centered iterative design process from learning engineering (Kessler, 2019) that enabled our evidence-based decision-making processes to select conference support technology platforms and create the conference infrastructure and workflows. We discuss a variety of data sources that informed our decision-making processes throughout the conference planning and actual event. We share assessment results and lessons learned that guide innovation in CTLs and can be applied to a range 
of educational development programs from large conferences like ours to small workshops and even one-to-one consultations.

Keywords: pedagogy conferences, scholarship of teaching and learning, online educational development, user experience, remote learning

What happens when your teaching center's signature event is turned on its head? When your annual 1-day conference-the one your center has nurtured for a decade from a 40-person extended morning workshop to a 400-person, 10-hour extravaganza that transforms the campus student center into a teaching center filled with hellos, hugs, and pedagogical aha! moments-is about to be shut down by a pandemic?

At George Mason University (GMU), where our unofficial motto is "innovation is our tradition," the Stearns Center for Teaching and Learning (Stearns Center) team rolled up our sleeves and started rebuilding. What began in March 2020 as a whoa! and then shifted into a series of what-ifs evolved 6 months later into a whole new level of teaching-and-learning outreach: a weeklong virtual conference with over 800 initial registrations, four new session types, three levels of session-host training, and not a single Zoom-bombing. In this article we outline some of our key experiences and takeaways, from the nittygritty of planning an online conference to some larger insights about how a user experience framework can guide planning for a range of center for teaching and learning (CTL) conferences, workshops, or smaller events.

\section{Early March 2020, Prologue:}

\section{A Teaching Conference at a Research University}

We had nothing special up our sleeves when we started this journey; our situation resembles that of many CTLs across the United States. 
GMU is the largest public institution in Virginia (U.S.). It is classified as research-intensive and has 3,608 instructional faculty (1,399 full time, 1,312 part time, and 897 graduate teaching assistants; GMU, 2019), serving about 38,000 students. The Stearns Center-formed 4 years earlier by combining the campus Center for Teaching and Faculty Excellence with an Office of Digital Learning-has a full-time staff of 14. Our Innovations in Teaching and Learning (ITL) conference has offered GMU faculty, staff, and graduate students the opportunity to share teaching strategies and research with one another annually since 2009. The ITL conference has three significant goals: to build a crossdisciplinary community around teaching and learning, to support instructors of all experience levels as they learn how to lead and join advanced conversations about pedagogy, and to celebrate teaching excellence by highlighting our award winners and faculty engaged in the scholarship of teaching and learning.

In recent years, the ITL conference has consisted of welcoming remarks from upper administrators, a keynote address and workshop by a nationally recognized speaker, a social breakfast and lunch, over 30 peer-reviewed breakout sessions (designed and led by GMU instructors, academic support office staff, and/or graduate students from across disciplines), and a final poster session and reception. The conference also produces an open-access peer-reviewed publication, Innovations in Teaching and Learning Conference Proceedings (https:// journals.gmu.edu/index.php/ITLCP/issue/archive), showcasing the peer-reviewed session descriptions and any supplementary session materials. The conference takes over a year to plan; it is led by the conference director (the lead author, who is assistant director in the Stearns Center) with logistical assistance from the center's events manager (a recently added staff position) and a student worker. Additional limited and short-term staff support is sourced on a volunteer basis from the center and other university departments and offices (e.g., Faculty Affairs, Office of Undergraduate Education).

In early March 2020, GMU faculty members who had volunteered as session reviewers had just submitted their evaluations of confer- 
ence proposals, the conference director was drafting a schedule, and the events manager was setting up registration and planning the food orders. Then came the COVID-19 pandemic. Like CTLs in most of the United States and around the world, we were suddenly neck-deep in providing emergency support for remote learning, but with the looming question, what should we do in September?

\section{Mid-March 2020, Lesson 1: Map Data Onto Priorities}

We faced an interlinked set of questions almost immediately: Should we proceed with or cancel the conference? If we proceeded, should we hope for clear sailing into a face-to-face fall semester or switch to a virtual conference? And how should we decide?

To start, we consulted local and national resources, mapping out our options, resources, goals, and challenges. First, we considered three commonly predicted scenarios: courses continuing to be offered fully online with most people working remotely; some combination of online and in-person classes and events; a return to courses and work primarily in person. Based on the available public health information about the virus at the time as well as concerns expressed by faculty and students locally and nationally, offering the conference in person was ruled out almost immediately-none of the data supported it.

Next, we surveyed the "alternative online event" landscape for solution trends. At our institution, new student and faculty orientations were already being planned as online-only events, which would help create a familiarity with online events by September (and give us some examples to learn from). We observed that other conferences that made a decision quickly and kept participants updated were more successful at keeping participants engaged. An online "something" thus seemed a viable logistical option.

Finally, we conducted a faculty needs assessment and drew on our tradition of user experience-based event design to determine what that online conference "something" would be, as well as when and 
how to announce the changes. We began by surveying the prospective presenters, explaining the situation, and inquiring whether they thought they would withdraw if we moved to an online format. We were pleasantly surprised when presenters overwhelmingly indicated that they would want to present in an online format.

At the same time, responses to our emergency course development programming made it clear that there would be ongoing professional development needs around course design, pedagogy, and teaching tools well into the fall semester. The ITL conference had the potential to be a critical link in the sustained educational development for these newly engaged faculty, many of whom were not connected to our center prior to the pandemic. Thus, the opportunities and needs balanced out the challenges of going forward with the conference online.

Through all these steps, we applied a user experience framework (Zarour \& Alharbi, 2017): What kinds of experiences did we most want presenters and participants to have at each stage of the conference organizing and event processes? (See Table 1.) Though CTL staff don't always think of faculty as "users" or our events as "platforms," our constant focus on meeting users' needs for functionality, efficiency, pleasure, and safety, in concert with our focus on the Stearns Center's larger priorities, helped us make decisions confidently even in the midst of so many unknown factors. CTL staff planning a single workshop in a non-pandemic year may not need so many bulwarks against uncertainty, but having a clear framework enables confident decisionmaking at any scale.

\section{Early April 2020, Lesson 2: Don't Dally}

For our large, complex conference, we needed to make decisions not just confidently but quickly, to ensure enough time to select, build, and refine the conference infrastructure system-the "platform" for learning. Particularly since our conference is run by and with a host of volunteers and newcomers, we needed time to build pathways and 
Table 1. Conference Support Platform Technologies Selection Criteria

\section{User experience design \\ framework principles}

Functionality/usability:

Products should work and

fulfill expected fundamental functions

\author{
Stearns Center ITL decision-making criteria
}

Platforms should map against our logistical needs. We need platforms to perform specific functions (e.g., capture participant information, display visual information, etc.) and have functional associated workflows within staff capacity limits.

Platforms should be easy to use across technologies. Platforms should be user friendly and visually appealing on phone as well as computer or tablet. Platforms should also be easily navigable and intuitive for organizing staff and accommodate internal workflows.

Platforms should be reasonably accessible. Platforms should provide or be able to integrate with accessibility technologies (e.g., captioning).

Platforms should include design elements or features that help participants feel connected and/or interact in ways similar to an inperson conference. Participants gain more if they are engaged. Additionally, our primary goal for the conference is community building-so this is a critical feature.

Efficiency: Products should minimize necessary user operations

Try to use the fewest platforms as well as platforms that synch and require the least number of clicks. Keep it simple. Organizers, presenters, and participants don't want to have to go between multiple platforms or too many steps to engage.

Pleasure: Products should prevent a bad first impression by enabling a user's natural handling and avoiding the feeling of inability

Platforms need to be visually appealing and user friendly, especially during a crisis. Participants are more likely to engage and return if they associate your event with positive emotions. Users (and organizers) are already running on low capacity due to the new stresses and elimination of non-essential workload during a crisis; they don't need the additional cognitive load of trying to figure out a new system or find things (like the Zoom link for a session)—it should be intuitive. If possible, leverage platforms that participants are already familiar with. By using something familiar, users and organizers can operate on auto-pilot, and the user demand and need for training/support from the organizers before and during the conference is likely reduced.

Comfort/safety: Products should prevent the feeling of insecurity

Platforms should offer users reasonable amounts of digital safety. Platforms should include mechanisms to protect user privacy and reduce trolling or social media bombing. Platforms should allow staff to be confident in data security.

Note. This table outlines a few key principles from user experience design frameworks (von Saucken et al., 2014; Zarour \& Alharbi, 2017) and the guiding selection criteria that we developed based on these principles. These criteria were applied while researching and selecting conference support platforms. We also selected our platform technologies within the constraints of budget and limited logistics staff capacity and workflows. 
support structures to enable a positive conference experience. By quickly making decisions, conference organizers can make large structural changes while there is still time to refine and implement them.

It was also vital that our plan support all aspects of a user's experience, from functionality to safety. In early April, session proposers were waiting anxiously for their overdue selection notifications. Since our conference planning approach aims to provide a positive and stress-free experience for participants, we knew that it was critical to decide and announce a comprehensive plan quickly. In retrospect, this sounds obvious, but in March and April 2020, choosing an unknown and possibly "lesser" conference venue felt agonizing. However, choosing early, purposefully, and comprehensively gave us the time we needed to be able to keep our principles at the forefront of all subsequent decisions.

\section{Mid-April 2020 and Beyond, Lesson 3: Communicate, Communicate, Communicate}

Communication is a crucial element to any CTL event, but our conference goals (of building community and increasing access), our specific situation (the newness and overwhelm of everything related to teaching in a pandemic), and our schedule (continuing to build the plane while we were flying it) made communication particularly crucial for us. We focused intensively on communication that would clarify everyone's new roles in the conference-essentially helping them imagine and prepare to take advantage of the revised user experience.

\section{Early Communication: Confirming the Schedule and Orienting Presenters}

We knew from previous experience that many faculty are largely unavailable over the summer, making it critical to inform presenters of the new format and protocols and have them confirm their intent to 
present by May. And even though the broader GMU community would leave for the summer uncertain about the overall university plans for the fall, our goal was that presenters would have enough information from us before summer to be able to effectively plan for our conference in September.

\section{Midsummer Communication:}

\section{Preparing for Interactive Online Sessions}

Since our twin goals of building community and enabling access for faculty at all levels were going to face additional challenges in online spaces, we needed to do more comprehensive outreach to help presenters and support staff prepare for an online conference experience. We had done versions of these outreach efforts before (e.g., guidelines and tip sheets), but we had also previously relied on academics' prior experiences with in-person conferences to help them navigate transitions and interactions fairly smoothly within a session.

Our presenter communication over the summer focused on strategies for building community through their online sessions. We required training sessions that modeled the user experience of a session: a tech host opened the training in the Zoom platform, and a session host welcomed them with an icebreaker activity. (Like DJs dreading "dead air" on the radio, we built in protocols to avoid awkward silence during the opening minutes in an online meeting as everyone waits for formal discussion to start.) We then shared "what to expect" information, walking them through registration, the session scheduling app (Sched, which allows attendees to view the full conference schedule and create personalized schedules), and how to access Zoom links as a presenter/host. We took extra time this year with our presenter development tips on how to bring participants into engaged learning activities (e.g., reflection moments; responding via shared chat, documents, or polls; virtual gallery walks) and integrated it with time for each presenter to practice related Zoom tool features with help from our events manager. Anecdotally, participants reported that the sessions were very helpful. 
We also knew that running an effective workshop, panel, or roundtable online requires navigating a host of multiple tasks at the same time: monitoring multiple input sources, sharing content orally with or without visual tools, and responding in real time across multiple outputs. To enable presenters to focus on their area of expertise, we trained staff members to schedule and launch each session and handle any tech issues (expanding on session hosts' prior face-to-face roles of introductions and timekeeping). We also walked them through code of conduct violation protocols and how to handle disruptive participants.

\section{Conference Month Communication:}

\section{Building Pathways to Participation}

As fall approached, we reached out to potential participants about how to register, how to access the conference schedule, and what to expect from a "typical" online session. We collaborated with our institution's technology help center to be able to direct any Zoom- and Sched-related access and troubleshooting questions to them. Finally, we did a range of direct recruiting: inviting deans and chairs individually to a panel about planning during a pandemic, for instance, or specifically inviting people who had participated in our new adjunct faculty workshops.

Our conference week communication was designed to support the whole user experience. Daily emails to all registered participants gave updates and directions, and our center's auto-reply was updated regularly to send an efficient message that gave helpful links and responses to FAQs. Behind the scenes, we used Microsoft Teams to set up our "mission control," to replicate the kind of "drop by" elements of a conference team managing user experience in a face-to-face site. Each tech host posted live status updates: Zoom session launched, host arrival, presenter arrival, session content started, session closed, and final attendance and poll results. As conference organizers, we could quickly look across concurrent sessions and step in where 
needed to support sessions. This process, like our other carefully planned communication efforts, saved a lot of time and energy and allowed participants to focus on their discussions about teaching and learning.

\section{Early May 2020, Lesson 4: \\ Be Selective About Technologies and Platforms}

Having a functional online conference system—or combination of systems-is essential. To offer the event online, we needed technology platforms that would be user friendly for participants, manageable for us to stand up, integrated with one another where possible, and not too overwhelming for everyone to learn. We identified Zoom as our best session venue platform. It offered the security options and stability at scale we needed, and by September we would have a site license and local tech support.

Of course, a venue, whether virtual or in person, is only one part of a conference. In April and May, we began sorting through all the other technologies that we could keep or adopt to support registration, scheduling, publicity, project management, evaluation, and archiving. We checked what other online conferences were using and took stock of resources we had used earlier: our WordPress-based website, our online journal for our conference proceedings, Sched, and our two past registration systems (TouchNet and Eventbrite). Then we rapidly tested those and other technologies across a period of 2 to 3 weeks for ease of use, fit, and budget. As we researched options and selected platforms, we relied on some key user experience frameworks (Zarour \& Alharbi, 2017) and guidelines (e.g., avoid the feeling of inability; von Saucken et al., 2014) to inform our platform selection criteria (see Table 1).

We eventually kept or adopted Constant Contact, Outlook, our WordPress-based website, and Twitter for external communication with participants; Microsoft Teams and Outlook for internal communi- 
cations; Eventbrite for registration; Sched for our conference program and participant access to Zoom links; Open Journal System (OJS) for our conference proceedings, which also doubled as a home for our On Demand sessions and repository for any supplementary materials associated with live sessions; and Qualtrics for evaluation. We were particularly pleased with the ways that Eventbrite integrated with Sched to create conference access and how Sched and Zoom provided options we could use to build inter-participant interaction and community.

Indeed, our front-facing choices were our most successful: data and observations indicate that we were able to support our participants by selecting platforms that were visually appealing and user friendly, accessible across desktop and mobile devices, well integrated, and supportive of our community building goals. The apps we chose also provided reasonable amounts of digital safety and privacy. However, our post-conference debriefs with our volunteers and student assistants identified the multiplicity of front-facing public sites and the number of communication pathways as an Achilles heel for our back-of-the-house operations. In our planning for next year, we are exploring ways to create a more streamlined operations plan with fewer moving parts or reimagined staff support roles.

\section{Mid-May 2020 and Beyond, Lesson 5: Be Bold in Reinventing Structures to Achieve Key Goals}

Moving a conference online creates new opportunities for participant interaction as well as new workload areas that need to be addressed and planned for. In an era of Zoom-bombing, we knew we had to develop new policies and statements about privacy, accessibility, and code of conduct; new tech support protocols; and guidelines for how to manage disruptive participants in order to work toward protecting participants' safety. But other improvements (see Table 2) came into focus more gradually. 

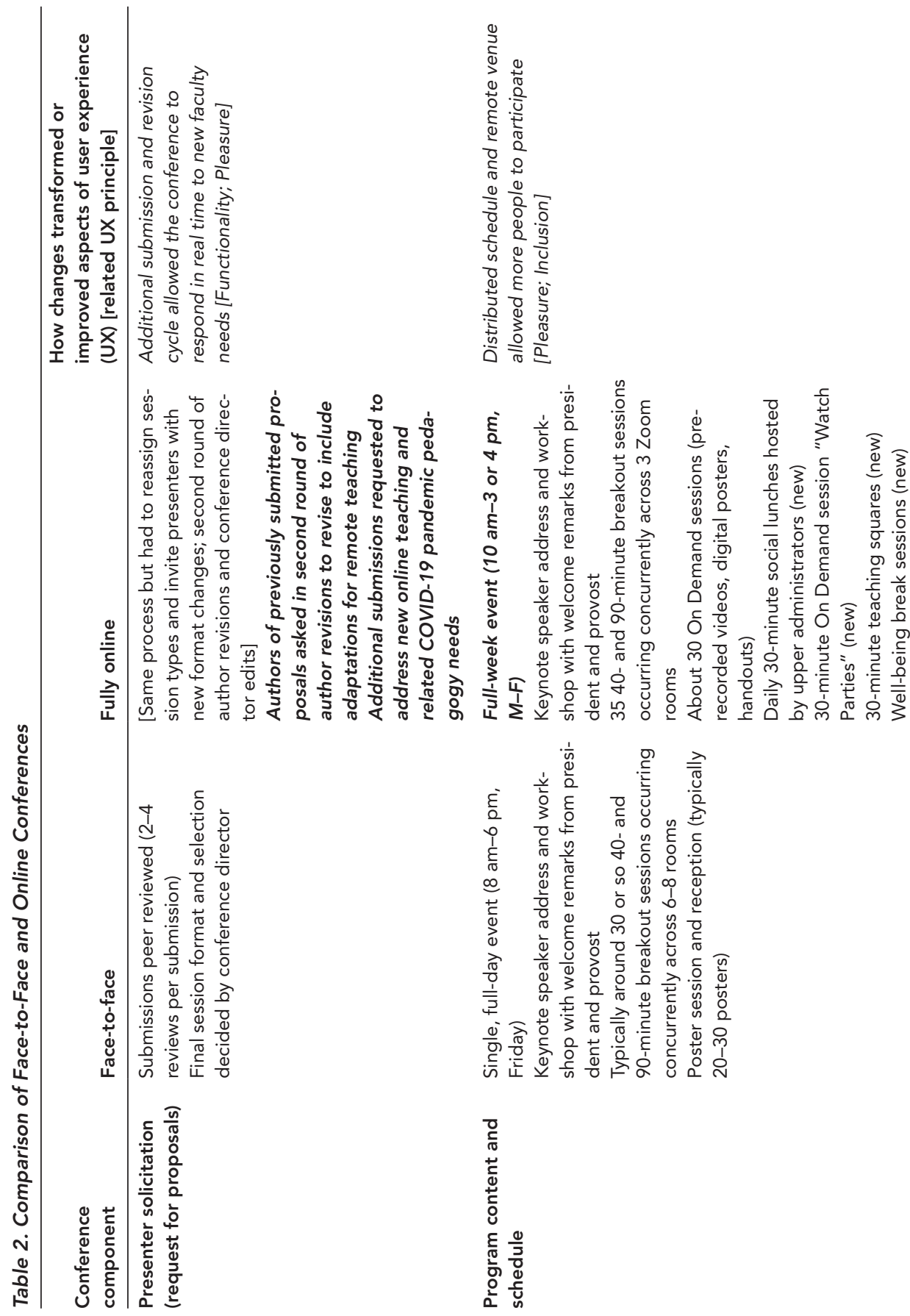

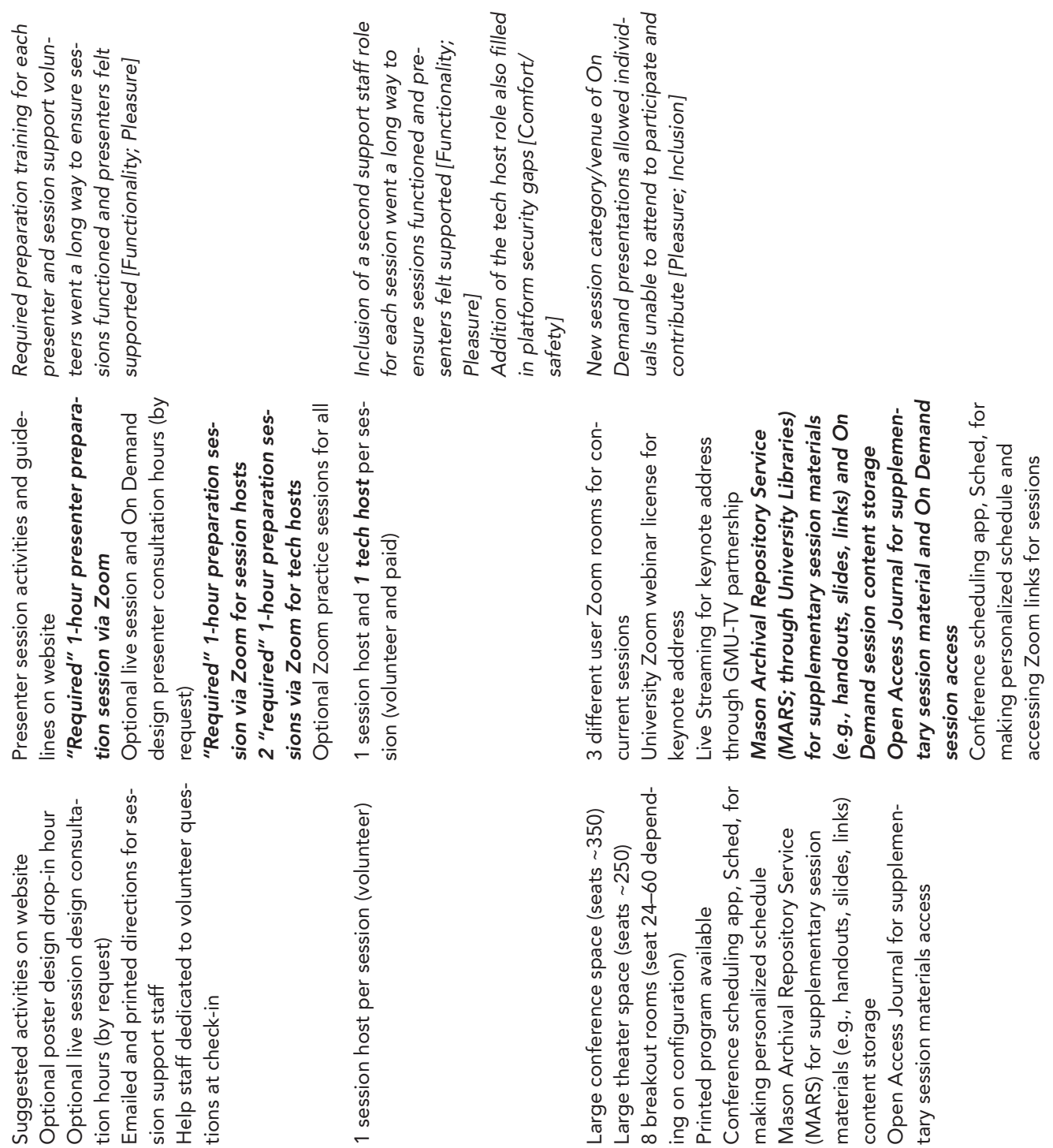

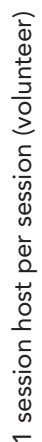
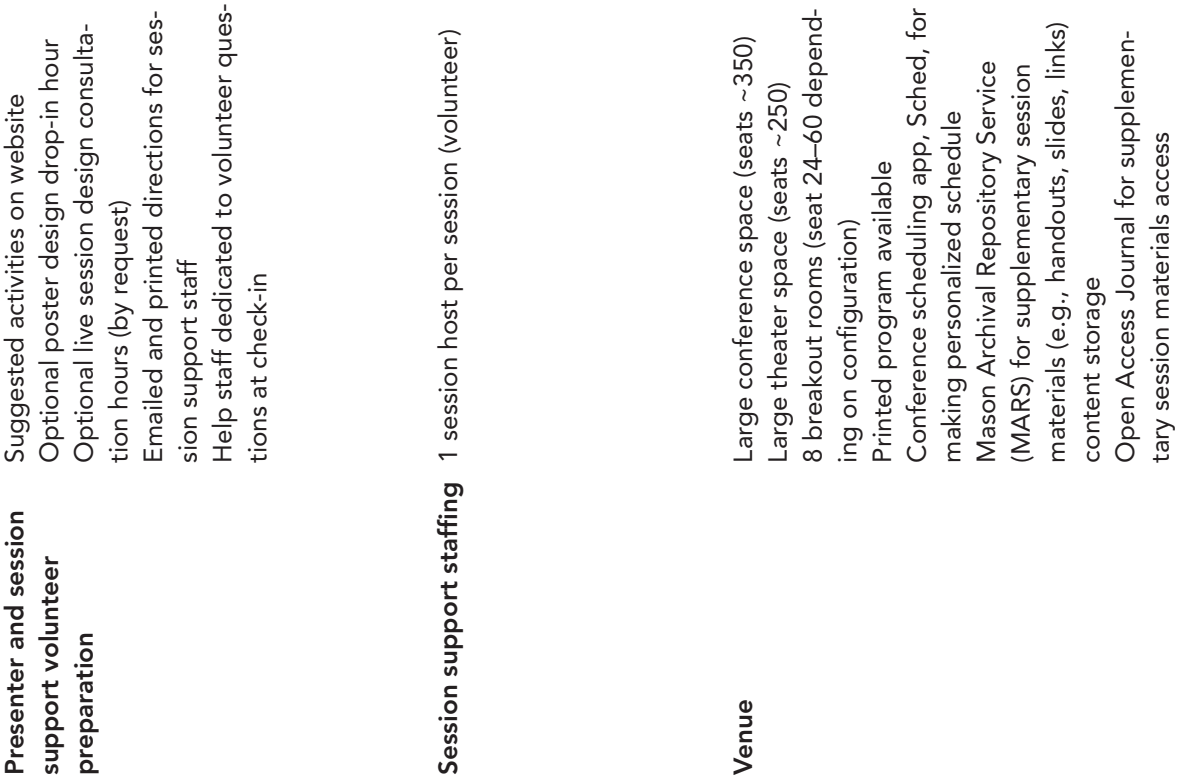

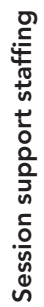

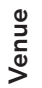



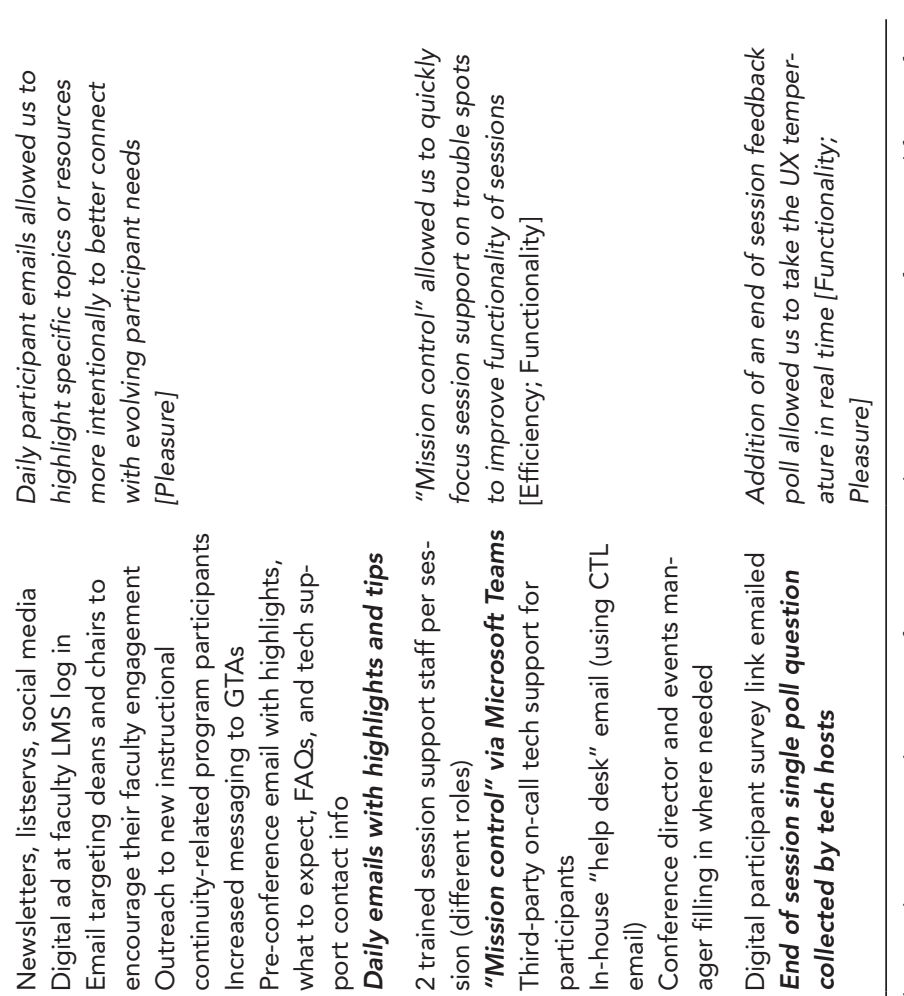
We decided early on that we would need to move away from a 1-day conference because Zoom fatigue was real: nobody would spend 10 straight hours looking at their peers through a computer window. We therefore chose to spread the conference program out over a full week, with sessions generally operating from 10 am to 3 $\mathrm{pm}$. This let us limit sessions to three at a time, which cut down on the number of session tech support people we needed to train and support.

Extending the schedule also increased conference accessibility: It allowed participants to "drop in" to parts of the conference even if they only had one or two spare hours in the week and made it possible for participants to attend more sessions overall if their schedules permitted. Our nearly $100 \%$ increase in registrations suggests that a good number of our constituents had been waiting for that kind of flexibility, even if not all of them could follow through on their intentions, and we are now looking for other ways to build those options into our smaller events and resources.

Like many conferences, ITL had long used volunteer session hosts to support presenters, but we used the summer to reimagine those roles, eventually coming to think of the sessions as learning ecosystems in which there were participants, presenters, and a session support team. Each session was assigned two volunteers, whom we recruited and trained: a tech host (who scheduled and launched the conference Zoom session, reported session status updates to mission control, managed breakout groups, and was prepared to address any unexpected issues like Zoom-bombing) and a session host (who welcomed participants to the conference, led a conference icebreaker activity, engaged participants in backchannel conversation in the chat, and closed out the session with next steps and a feedback poll). Like any carefully scaffolded learning opportunity, the intensive prior planning looked a bit like overkill at first glance-would there still be room for presenters' own personalities and audience spontaneity?-but in practice, we found that the extra personnel and protocols became "features" of our conference system that increased participants' abil- 
ity to focus on the session and not the trappings or awkwardness of the venue.

Finally, as we moved through summer, it became clear that we might take further advantage of silver linings and experiment with brand-new session structures and types. Upper-level administrators who were impossible to schedule for 90 minutes in person on a Friday could be invited to a remote 30 -minute lunch conversation; contingent faculty who worked during live session times would be able to view the On Demand sessions; static posters could become more engagingly narrated videos; shared documents created a permanent "trace" of participants' small group brainstorming that we could disseminate widely (Table 2 maps out our most valuable adaptations). Although our experiments were initially driven by necessity, our successes have given us reason to examine other ways we can innovate without losing sight of key goals or constituent needs.

\section{June 2020 and Beyond, Lesson 6:}

Stay Reflective and Flexible

We adopted an iterative design approach to planning the conference experience (Kessler, 2019), which provided ongoing opportunities for evidence-based feedback. As we set up our technology platforms, we conducted user experience tests (acting as participant users and asking colleagues to act as participant users) in which we identified potential challenges for participant users. As part of our staff meetings, we engaged in self-reflective group check-ins about how technologies and associated workflow systems were working from a planning perspective. During the event, as noted, we utilized a virtual mission control center that allowed the conference director and events manager to monitor the successful launch and completion of sessions. By building iterative user testing into all stages, we were able not just to lower frustration and increase efficiency but also to create even more accessible and rewarding experiences than in prior years. 


\section{October 2020, Epilogue: "The first Zoom meeting where everything worked"}

Ultimately, our success was measured by functionality of the conference sessions and participant responses before, during, and after attending the sessions. Anecdotally, many session hosts and participants_including a faculty member who noted it was "the first Zoom meeting where everything worked"-remarked how smoothly everything ran and how they enjoyed the sessions. Conference feedback survey data ( $n=93$ ) was very positive as well, with $88 \%$ reporting it was helpful or very helpful, 93\% reporting they would recommend it to a colleague, and $95 \%$ reporting they would attend again. In terms of value, the majority of respondents reported that participating in the ITL conference increased their confidence as an instructor (81\%), built their teaching skills (91\%), increased their connection to others (88\%), and allowed them an opportunity to meet other people (90\%). Interestingly, only $9 \%$ reported that they would attend again, but only if in person, suggesting that the online format was not a prohibitive factor for participating in future events. These numbers parallel previous Stearns Center events and reassure us that overall, our online model was a logistical success.

More than that, our conference was a faculty development success-and a positive user experience-in line with our key principles. In the end our participation numbers did not match our high registration count: 451 unique users checked in across Zoom sessions, and 43 anonymous users live-streamed the keynote address outside of Zoom. (We are unable to determine how many users have accessed the On Demand resources.) But that participation level was still almost $15 \%$ higher than last year and held steady throughout the week, which already has us thinking we won't ever go back to an in-person 1-day-only model. Our pre-planning helped presenters in each session give opportunities for participants to engage with one another, supporting them in building community and, through guided activities, creating the "practical takeaways" that participants tell us they value. 
Our mission control center tactic ensured seamless session logistics and provided us with additional data about who attended what and how they felt, which we're looking forward to analyzing to help extend the future reach of the Stearns Center. Lastly, instead of an archive primarily of PDFs of posters, we have a series of multimedia learning objects that we're looking forward to curating and repurposing in the coming months.

Ten months later, though we are still debriefing the conference, we're confident that we didn't just "make lemonade out of lemons," surviving through a crisis. Instead, by making some early, user-centered, imaginative-and-yet-data-based decisions, we planted a whole new tree that will bear fruit well into the future.

\section{Biographies}

Laura A. Lukes is an Assistant Director for Teaching Excellence in the Stearns Center for Teaching and Learning and is affiliated with Atmospheric, Oceanic, and Earth Sciences at George Mason University. Her work appears in the Journal of Geoscience Education, Journal of College Science Teaching, and SPUR. She is an Albert Einstein Distinguished Educator Fellow (2010-2011) and Fellow of the Geological Society of America. She has served as the Director for the Innovations in Teaching and Learning conference since 2014.

E. Shelley Reid is Co-director of the Stearns Center for Teaching and Learning and an Associate Professor of English at George Mason University. Her work on teacher preparation, mentoring, and writing education appears in Composition Studies, College Composition and Communication, Pedagogy, Writing Program Administration, and Writing Spaces. 


\section{Acknowledgments}

Thank you to Ashley Joiner, MA, Events Manager in the Stearns Center for Teaching and Learning at George Mason University, for her contributions to the conference planning and logistical support during the conference.

\section{References}

George Mason University. (2019, Fall). Faculty and staff by division. https://oi ep.gmu.edu/data-analytics-research/human-resources/faculty-staff-by-divi sion/

Kessler, A. (2019). What is learning engineering? IEEE ICICLE Conference Proceedings. https://sagroups.ieee.org/icicle/learning-engineering-process/

von Saucken, C., Lachner, F., \& Lindemann, U. (2014). Principles for user experience: What we can learn from bad examples. Proceedings of the 5th Kanesi Engineering and Emotion Research Conference, Linköping, Sweden, June 11-13. https://ep.liu.se/ecp/article. asp?issue=100\&volume=\&a rticle $=110$

Zarour, M., \& Alharbi, M. (2017). User experience framework that combines aspects, dimensions, and measurement methods, Cogent Engineering, 4(1). https://doi.org/10.1080/23311916.2017.1421006

To Improve the Academy • Vol. 39, No. 3 • Spring 2021 
